\title{
Role of histopathologic and phenotypic assessment in the development and validation of optical diagnostic devices for head and neck mucosal lesions
} Adel K El-Naggar

Address: Division of Pathology, UT M.D. Anderson Cancer Center, Texas, USA

from Ist Scientific Meeting of the Head and Neck Optical Diagnostics Society London, UK. 14 March 2009

Published: 28 July 2009

Head \& Neck Oncology 2009, I(SuppI I):O9 doi:I0.I I86/I758-3284-I-SI-O9

This abstract is available from: http://www.headandneckoncology.org/content/I/SI/O9

(C) 2009 El-Naggar; licensee BioMed Central Ltd.

Head and neck squamous carcinoma, including the oral cavity, is the sixth most common cancer worldwide with approximately 270,000 new oral cavity tumours per year. Unfortunately, the majority of these tumours present in late stage with the attended functional, psychological and economic costs on their victims. It's clearly evident that screening and early detection of the cancer and its early precursors have the potential to reduce the morbidity and mortality of this disease. In that context, current oral examination methods including incandescent light or toluidine blue, reflectance visualization and illumination with chemiluminescent light source, are largely subjective, dependent on the experience of the examiner and are considered in-effective tools in primary care settings.

Autofluorescence imaging has recently been shown to improve the detection of premalignant and malignant oral lesions. This method is based on the illumination in the absorption of tissue fluorophore molecules (NADH and FAD in the epithelial layer and collagen, and elastin in the stroma) in ultraviolet visible spectrum leading to the emission of lower energy photon that can be detected as fluorescence from the oral surface mucosa. Studies of these methods in normal oral mucosa have shown increased green fluorescence in comparison to neoplastic lesions upon ultraviolet (UV) or near UV light source. The histopathologic manifestations and heterogeneity of oral squamous lesions and the confounding factors for the validation and the clinical applications of autofluoresence imaging will be presented and discussed. 